\title{
On the thermally induced interfacial delamination of a segmented coating
}

\author{
Xuejun Chen ${ }^{\mathrm{a}, \mathrm{b}, *}$, Guangnan Chen ${ }^{\mathrm{b}}$, Kun Zhang ${ }^{\mathrm{b}}$, Gengxing Luo ${ }^{\mathrm{b}}$, Jinghua Xiao ${ }^{\mathrm{b}}$ \\ ${ }^{a}$ School of Applied Sciences, University of Science and Technology Beijing, No. 30 Xueyuan Road, Beijing 100083, PR China \\ ${ }^{\mathrm{b}}$ Institute of Mechanics, Chinese Academy of Sciences, Beijing 100080, PR China
}

Received 4 July 2007; accepted in revised form 19 October 2007

Available online 4 November 2007

\begin{abstract}
The thermally induced interfacial delamination problem of a segmented coating is investigated using finite element method (FEM). The coating-substrate system, modeled as a coated semi-infinite medium with periodic segmentation cracks within coating, is assumed to be exposed to convective cooling from surface. The failure criterion based on the interfacial fracture toughness is adopted, in which the energy release rate for an interface crack is considered to be the driving force for interfacial delamination extension. The results confirm that a segmented coating has higher delamination resistance than an intact one under the same thermal transients, as the segmentation crack spacing is smaller than a critical value. Based on dimensional analysis, sensitivity analyses of the crack driving force are also obtained as a function of various dimensionless parameters such as time, convection severity and material constants. These results may provide some helpful references for the integrity of coating-substrate systems under thermal loading.
\end{abstract}

(C) 2007 Elsevier B.V. All rights reserved.

Keywords: Interfacial delamination; Thermal loading; Segmentation crack; Dimensional analysis; Finite element method

\section{Introduction}

In many modern applications, protective coatings are deposited onto the load-bearing substrate to improve a certain surface characteristics, e.g. thermal, corrosion and wear resistance $[1,2]$. However, thermal conditions which arise during heating or cooling of the coating-substrate system can result in very high strain mismatch between the coating and substrate. If the thermal transient is severe enough, interfacial delamination may occur, as shown in Fig. 1. This poses a major threat to the safety of the entire thermo-mechanical system since it will lead to the final removal of coating.

Due to its practical importance, the interfacial delamination mechanics of coating-substrate system has been the subject of considerable research [3-7]. In order to determine the severity of thermal conditions that a given coating-substrate system can withstand, two alternative fail-safe criteria have been utilized, one based on interfacial adhesion strength and the other on

\footnotetext{
* Corresponding author. School of Applied Sciences, University of Science and Technology Beijing, No. 30 Xueyuan Road, Beijing 100083, PR China. Tel.: +8610 62332985; fax: +861062327283.

E-mail address: chenxuejuncas@sohu.com (X. Chen).
}

interfacial fracture toughness. In general, the latter treatment deals with the cases more close to the practical situations in which pre-existing interface cracks are assumed to exist. In parallel efforts, various coating designs are proposed to enhance the durability of coating such as functionally gradient coating, duplex coating as well as multilayer coating [8-10]. The concept of these designs takes foot on an intact coating and the main purpose is to alleviate the mismatch stress level across the interface.

Recently a new concept of coating design, i.e. the segmented coating, has been successfully developed to improve the durability of coating in a fluctuating thermal environment [11-13]. Herein the segmented coating refers to a coating with many segmentation cracks which run from the surface of coating toward the interface of coating/substrate with the penetration depths comparable to the coating thickness. Fig. 2a and $b$ shows the development of periodic segmentation cracks within electroplated chromium coating by the hybrid technique of laser pre-quenching substrate plus post-deposition. Firstly, as shown in Fig. 2a, multiple micro-surface-cracks are initiated corresponding to the discrete laser quenching pattern, i.e. one micro-surface-crack occurs between two adjacent pre-quenched regions. When the required vacuum annealing is performed to 


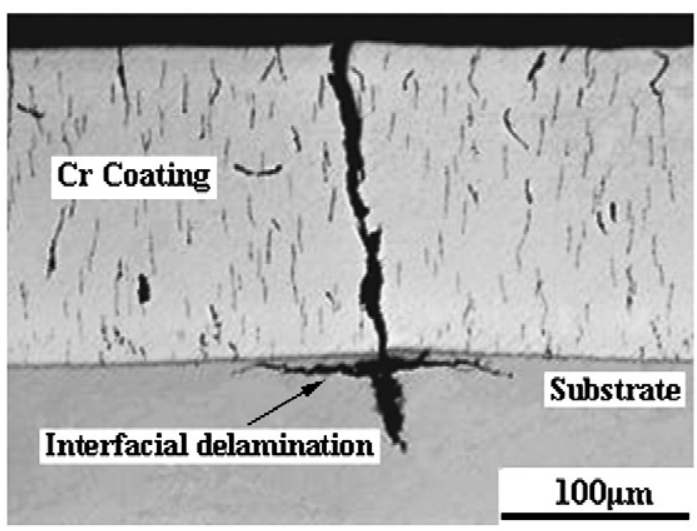

Fig. 1. Interfacial delamination of a $\mathrm{Cr}$ coating/Steel substrate subjected to thermal loading.

dehydrogenate, these cracks become wider and run toward the interface of the coating-substrate system. Finally, periodic segmentation cracks are developed after few thermal cycles, as shown in Fig. 2b. It should be noted that the density of the formed segmentation cracks can be controlled by adjusting the hybrid processing parameters. The detailed preparation process on laser-quenching of the steel and chromium coating electroplating can be found in Ref. [13].

Experimental results [13] demonstrated that the segmented coating could largely survive the intact coating, i.e., the former can bear more thermal cycles prior to failure. Actually, the similar beneficial effect of a pre-cracked coating has been experimentally observed for plasma sprayed Thermal Barrier Coatings (TBC) in previous literature [14,15], in which it is argued that in-plane compliance of the pre-cracked coating can be increased and therefore, the fatal interfacial debonding can be delayed. The objective of this paper was to reveal its mechanism from the standpoint of fracture mechanics, in which the energy release rate of an interface crack is the driving force for interfacial delamination propagation. Moreover, to provide some useful references for coating-substrate design under thermal

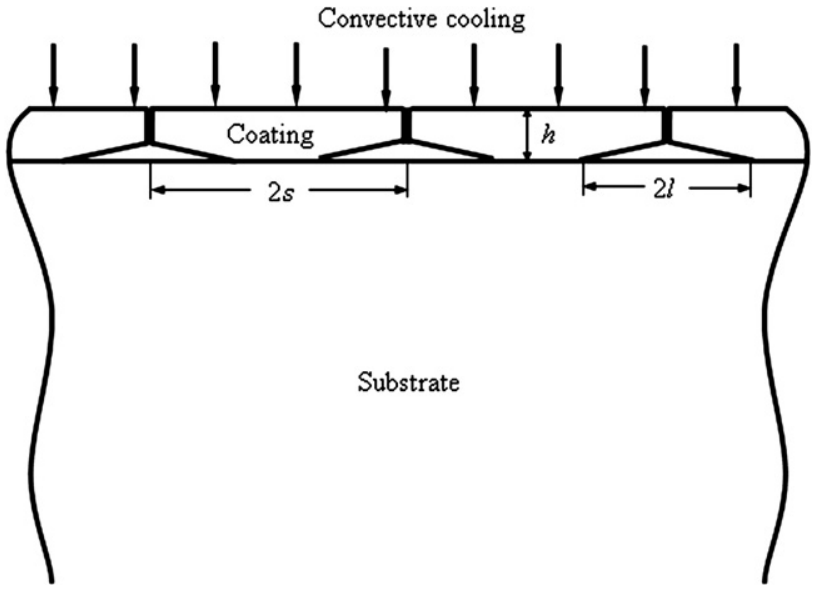

Fig. 3. The segmented coating-substrate system subjected to convective cooling from surface.

loading, the sensitivity analysis of this crack driving force is performed to correlate it with certain important parameters such as time, thermal severity and material constants.

In solving the problem, both coating and substrate materials are assumed to be homogeneous, isotropic and linear-elastic, the thermo-mechanical constants are independent of temperature, and the thermo-elastic coupling effects and inertia effects are negligible. The last assumption, which simplifies the analysis of the problem quite considerably, was justified by previous work on dynamic thermo-elasticity [16,17]. All the calculations are performed using the finite element method (FEM).

\section{Problem formulation}

Since a substrate is commonly much thicker than a coating, the segmented coating-substrate system is modeled, in Fig. 3, as a coated semi-infinite medium with uniformly spaced segmentation cracks within the coating. The coating thickness is denoted by $h$. Although the segmentation cracks may further

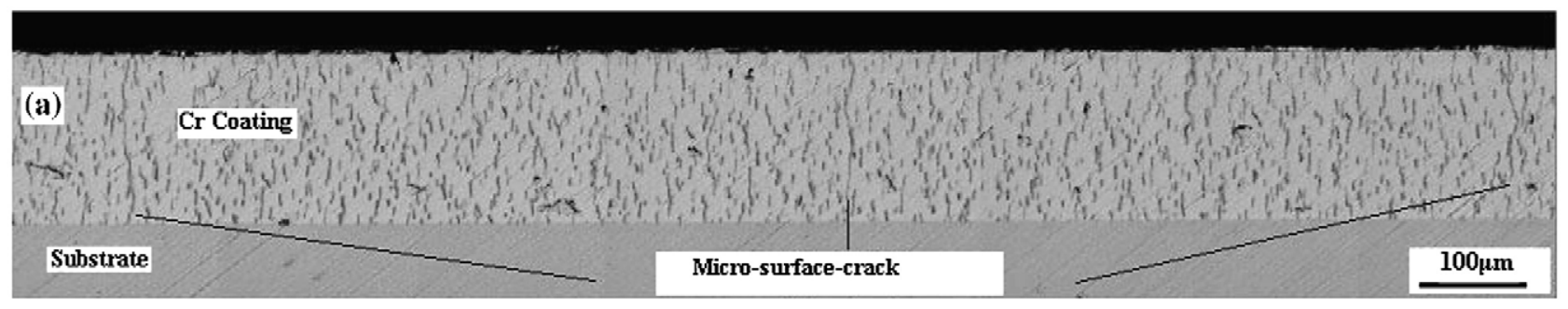

(b)

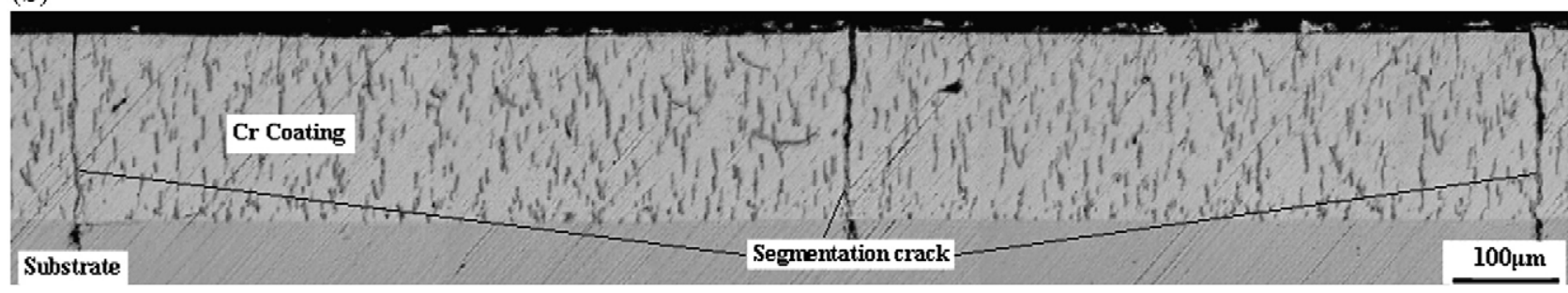

Fig. 2. The profile of: (a) initiated micro-surface-cracks and (b) periodic segmentation cracks by the hybrid technique of laser pre-quenching substrate plus postdeposition. 
penetrate into substrate, the corresponding depth within the substrate is usually much smaller than the coating thickness and experimental results [13] show this negligible depth almost remains up to the final delamination of coating. Therefore, multiple segmentation cracks, with penetration depth $h$ and crack spacing $2 \mathrm{~s}$, have been modeled, omitting the negligible segment within the substrate. To simulate the realistic situation as in Fig. 1, interfacial cracks, each length of $2 l$, are assumed to be present. The coated medium is initially at a constant temperature $T_{0}$. At time $t=0$, the surface is subjected to convective cooling with the heat transfer coefficient given by $H$ and the ambient temperature maintained at $T_{a}$.

Because of periodicity, we only need to consider a representative region of width $s$ adjacent to one segmentation crack. For this region, the coordinate axes are chosen such that the $x$-axis coincides with the interface and $y$-axis along the corresponding segmentation crack, as shown in Fig. 4. The transient temperature distribution is governed by the ordinary heat diffusion equations, which read

$\nabla^{2} \theta_{\gamma}(x, y, t)=\frac{1}{D_{\gamma}} \frac{\partial \theta_{\gamma}(x, y, t)}{\partial t} \quad(\gamma=1,2)$

where temperature deviations $\theta_{\gamma}(x, y, t)$ are defined by

$\theta_{\gamma}(x, y, t)=T_{\gamma}(x, y, t)-T_{0} \quad(\gamma=1,2)$

$\theta_{0}=T_{a}-T_{0}$

The subscripts 1 and 2 refer to coating and substrate, respectively. The thermal diffusivity $D_{\gamma}$ is given by $D_{\gamma}=k_{\gamma} / \rho_{\gamma} c_{\gamma}$,
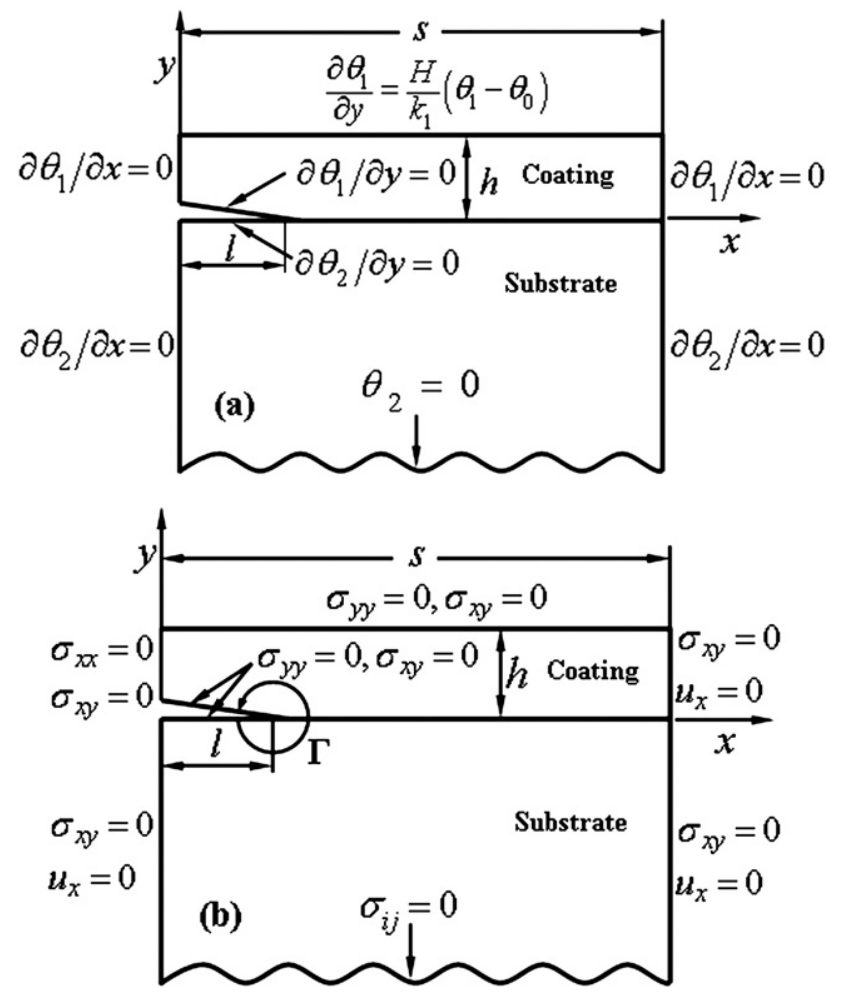

Fig. 4. (a) The thermal boundary conditions and (b) mechanical boundary conditions used in the representative region. where $k_{\gamma}$ is the material thermal conductivity, $\rho_{\gamma}$ the density and $c_{\gamma}$ the specific heat. In the case of convective cooling, $\theta_{0}<0$.

The initial conditions are given by

$\theta_{1}(x, y, 0)=0, \quad 0 \leq x \leq s ; 0 \leq y \leq h$

$\theta_{2}(x, y, 0)=0, \quad 0 \leq x \leq s ;-\infty<y \leq 0$.

The temperature and heat flux are assumed to be continuous at the bonded interface, neglecting contact thermal resistance. The interface crack is assumed perfectly insulated which would provide an upper bound solution for the subsequent energy release rate for it [18]. For convenience, thermal boundary conditions are summarized in Fig. 4a.

As for thermal stresses calculation, the equilibrium equation

$\partial \sigma_{i j}(x, y, t) / \partial x_{j}=0$

holds for $x_{i}=x, y$. The stress tensor is given by

$$
\begin{aligned}
\sigma_{\mathrm{ij}}(x, y, t)= & \frac{-E}{1-2 v} \alpha \theta(x, y, t) \delta_{i j} \\
& +\frac{E}{1+v}\left[\varepsilon_{\mathrm{ij}}(x, y, t)+\frac{v}{1-2 v} \delta_{\mathrm{ij}} \varepsilon_{\mathrm{kk}}(x, y, t)\right]
\end{aligned}
$$

where $\varepsilon_{\mathrm{ij}}(x, y, t)=\frac{1}{2}\left[\partial u_{i}(x, y, t) / \partial x_{j}+\partial u_{j}(x, y, t) / \partial x_{j}\right]$ represents the strain tensor, $u_{i}(x, y, t)$ the displacement vector and $\delta_{\mathrm{ij}}$ Kronecker delta. The symbols $\alpha, E, v$ denote coefficient of thermal expansion (CTE), Young's modulus and Poisson's ratio, respectively. It should be emphasized that Eqs. (4) and (5) are valid for both the coating and substrate and that the Einstein summation rule is indicated when the suffixes in a single term are repeated.

At any instant across the bonded interface, the deformation should meet the requirement of continuity in displacements and both interfacial normal stress and interfacial shear stress are identical for either coating or substrate. The problem is solved under the assumption of plane strain conditions with mechanical boundary for thermal stress calculations summarized in Fig. $4 b$.

As the result of thermal stresses, at any instant the stress intensity builds up around the interfacial crack tip. For a bimaterial medium, the stress intensity factor is intrinsically mixmoded with both an opening mode $K_{1}$ and a shearing mode $K_{2}$, i.e., the interfacial crack propagation is both tensile and sheardriven [3]. Here the energy release rate for an interface crack is adopted to characterize the driving force, which is related to the complex stress intensity factor, $K(t)=K_{1}(t)+i K_{2}(t)$, by [3]

$$
G(t)=\frac{|K(t)|^{2}}{\cosh ^{2}(\pi \varepsilon) E^{*}}
$$

where $\frac{1}{E^{*}} \frac{1}{2}\left(\frac{1}{\bar{E} 1}+\frac{1}{\overline{E 2}}\right), \bar{E}=E_{\gamma} /\left(1-v_{\gamma}^{2}\right) \quad(\gamma=1,2)$. The expression includes the bi-material constant, $\varepsilon$, defined as

$$
\varepsilon=\frac{1}{2 \pi} \ln \left(\frac{1-\beta_{D}}{1+\beta_{D}}\right)
$$


with $\beta_{D}$ being second Dundurs' parameters, which, in turn, is defined as

$$
\beta_{D}=\left[\mu_{1} / \mu_{2}\left(\lambda_{1}-1\right)-\left(\lambda_{2}-1\right)\right] /\left[\mu_{1} / \mu_{2}\left(\lambda_{1}+1\right)+\left(\lambda_{2}+1\right)\right]
$$

where $\mu_{\gamma}=\frac{E_{\gamma}}{2\left(1+v_{\gamma}\right)}$ is shear modulus and $\lambda_{\gamma}=3-4 v_{\gamma}(\gamma=1,2)$.

Since the above transient mixed boundary problem is analytically intractable and therefore will be solved using FEM. Various finite element methodologies, such as crack flank displacement method and J-integral method [18-20], have been developed to evaluate the energy release rate for the interfacial crack under thermal loading. In the current computation, the latter is chosen. At any time instant, the path-independent J-integral can be extended to bi-material bodies [21], and is defined as

$J(t)=\int_{\Gamma}\left(W(x, y, t) n_{x}-\sigma_{j k}(x, y, t) n_{k} \frac{\partial u_{j}(x, y, t)}{\partial x}\right) d \Gamma$

where $\Gamma$ is a path of arbitrary contour which begins at any point on lower crack flank, encircle the tip, and ends at any point on the upper crack surface (See Fig. $4 b$ ). $W$ is the strain energy density, $u_{i}$ the displacement vector, $n_{i}$ the unit outward normal of $\Gamma$.

The magnitude of complex stress intensity factor correlates with the J-integral value by [18]

$J(t)=\frac{\Lambda_{1}+\Lambda_{2}}{16 \cosh ^{2}(\pi \varepsilon)}|K(t)|^{2}$

where $\Lambda_{\gamma}=4\left(1-v_{\gamma}\right) / \mu_{\gamma}$. From Eqs. (6) and (10), the relation between $J$ and $G$ reads

$G(t)=\frac{16 J(t)}{E^{*}}\left(\Lambda_{1}+\Lambda_{2}\right)$.

\section{Solution procedures}

\subsection{Dimensional analysis}

Before the solution to the above problem is known, dimensional analysis can be carried out to reveal the scaling relationships of this problem. The temperature deviations $\theta_{\gamma}(\gamma=1,2)$ must be functions of all the independent governing parameters, i.e., temporal and spatial coordinates $t, x$ and $y$; characteristic geometrical parameters $h, s$ and $l$; thermo-physical constants $D_{1}, D_{2}, k_{1}, k_{2}, H$ and ambient temperature deviations $\theta_{0}$

$\theta_{\gamma}(x, y, t)=\theta_{\gamma}\left(x, y, t ; h, s, l ; D_{1}, D_{2}, k_{1}, k_{2}, H ; \theta_{0}\right)$.

Among the twelve governing parameters, four of them, namely $h, D_{1}, k_{1}$ and $\theta_{0}$, have independent dimensions. The dimensions of $\theta_{\gamma}, x, y, t, s, l, D_{2}, k_{2}$ and $H$ are given by

$$
\begin{aligned}
& {\left[\theta_{\gamma}\right]=\left[\theta_{0}\right] \quad[x]=[y]=[h] \quad[t]=\left[D_{1}\right]^{-1}[h]^{2} \quad[s]=[h]} \\
& {[l]=[h] \quad\left[D_{2}\right]=\left[D_{1}\right] \quad\left[k_{2}\right]=\left[k_{1}\right][H]=\left[k_{1}\right][h]^{-1}}
\end{aligned}
$$

On applying $\Pi$-theorem in dimensional analysis [22], we obtain

$\frac{\theta_{\gamma}}{\theta_{0}}=\Pi_{\theta}\left(\frac{x}{h}, \frac{y}{h}, \frac{D_{1} t}{h^{2}} ; \frac{s}{h}, \frac{l}{h} ; \frac{D_{2}}{D_{1}}, \frac{k_{2}}{k_{1}}, \frac{H h}{k_{1}}\right)$.

The following dimensionless quantities are then naturally defined as

$\bar{x}=\frac{x}{h} \quad \bar{y}=\frac{y}{h} \quad \bar{s}=\frac{s}{h} \quad \bar{l}=\frac{l}{h}$

$\bar{D}=\frac{D_{2}}{D_{1}} \quad \bar{k}=\frac{k_{2}}{k_{1}} \quad B i=\frac{H h}{k_{1}} \quad F o=\frac{D_{1} t}{h^{2}}$

Based on the above dimensional analysis, the dimensionless quantity $\theta_{\gamma} / \theta_{0}$ on the left side of Eq. (14) is determined only by eight bracketed dimensionless quantities on the right side, in which $\mathrm{Bi}$ and Fo are similar to conventional Biot number and Fourier number, respectively. The most severe thermal shock case corresponds to a sudden cooling, i.e., $\mathrm{Bi} \rightarrow \infty$.

The thermal stress will be induced as the consequence of the transient temperature field in operation. In terms of timedependent thermal stress, the energy release rate for interfacial crack is also time-dependent, which is the function of the following quantities

$G(t)=G\left(t, \theta_{0} ; h, l, s ; D_{1}, D_{2}, k_{1}, k_{2}, H ; E_{1}, E_{2}, v_{1}, v_{2}, \alpha_{1}, \alpha_{2}\right)$.

On applying $\Pi$-theorem again, the energy release rate can be of the dimensionless form

$\frac{G}{E_{1}\left(\alpha_{1} \theta_{0}\right)^{2} h}=\Pi_{G}\left(\frac{D_{1} t}{h^{2}} ; \frac{l}{h}, \frac{s}{h} ; \frac{D_{2}}{D_{1}}, \frac{k_{2}}{k_{1}}, \frac{H h}{k_{1}} ; \frac{E_{2}}{E_{1}}, \frac{v_{2}}{v_{1}}, \frac{\alpha_{2}}{\alpha_{1}}\right)$.

As can be shown by Eq. (17), three additional dimensionless material properties, $\bar{E}=E_{2} / E_{1}, \bar{v}=v_{2} / v_{1}$ and $\bar{\alpha}=\alpha_{2} / \alpha_{1}$ should appear automatically in energy release rate analysis, which measure the ratios of Young's modulus, Poisson's ratio and coefficient of thermal expansion (CTE), respectively. It should be noted that ratios of Young's modus and Poisson's ratio as shown here can correlate with the well-known Dundur's parameters (For example, the first Dundur's parameter $\alpha_{D}$ is simply connected to ratio of Young's modulus: $\alpha_{D}=(1-\bar{E}) /$ $(1+\bar{E})[3])$.

With the help of the above dimensional analysis, sensitivity analysis for temperature, interfacial crack energy release rate can be conveniently performed, which will be detailed in Section 4.

\subsection{Finite element model}

In this paper, FEM has been used to calculate both temperature and interfacial crack energy release rate. The thickness of substrate is taken as $200 \mathrm{~h}$ to capture the essence of infinity. Eight-node quadrilateral elements are used here for both temperature and displacement calculations. The temperature distribution has been computed using the commercial finite element package ANSYS [23]. In order to obtain accurate 


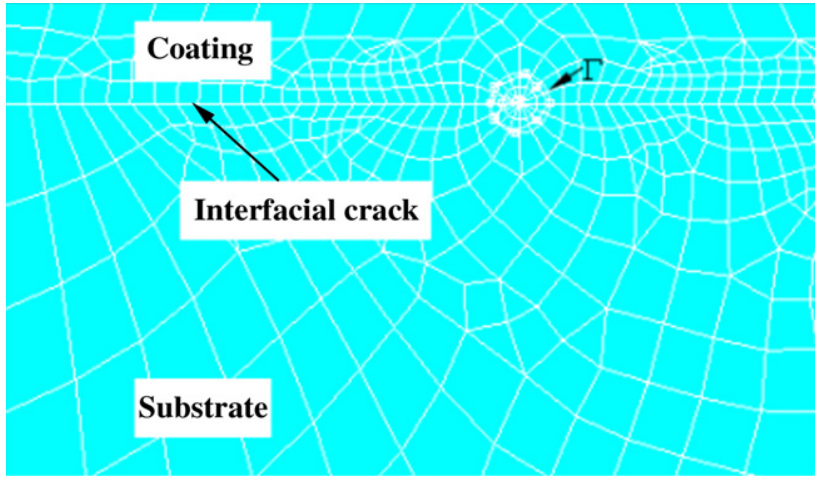

Fig. 5. Typical finite element meshes around the interfacial crack tip. $\Gamma$ is a path of arbitrary contour which begins at any point on lower crack flank, encircle the tip, and ends at any point on the upper crack surface.

results, careful element meshing is necessary to assure a reasonable aspect ratio of the elements, and particularly element refinements should be done in the vicinity of the crack tip and the interface of coating and substrate.

With the transient temperature distribution known, it is easy to determine displacement and thermal stresses by the classical finite element formulation. It should be noted that, in finite element model, the bottom of the substrate is constrained against both vertical and horizontal displacement. The detailed mesh breakdowns around the crack tip are schematically illustrated in Fig. 5. ANSYS finite element codes have been used again for the evaluation of interfacial crack energy release rate $\mathrm{G}$. The code evaluates $\mathrm{G}$ on the basis of path-independent J-integral through Eq. (11). In order to minimize the computational inaccuracy, the ultimate value of J-integral is averaged over those of three different contours.

\section{Numerical results and discussions}

As an example for the thermally induced interfacial delamination problem of a segmented coating described in the previous sections, a material pair, corresponding to a chromium coating electroplated onto a structural steel substrate $30 \mathrm{CrNi2}-$ MoVA, has been considered. Since the problem is formulated in terms of dimensionless quantities, it is sufficient to consider only the ratios of material constants shown in Table 1. Unless otherwise stated, the data in our calculations are taken from Table 1. All the quantities of interest are normalized in the same forms as those in Section 3.1.

\subsection{Results of temperature field}

Some results concerning transient temperature distribution are shown in Figs. 6 and 7. In order to avoid confusion, a dash line is embedded in both figures to indicate the "physical" low

Table 1

The dimensionless quantities used in the example [24]

\begin{tabular}{lllll}
\hline$D_{2} / D_{1}$ & $k_{2} / k_{1}$ & $E_{2} / E_{1}$ & $v_{2} / v_{1}$ & $\alpha_{2} / \alpha_{1}$ \\
\hline 0.293 & 0.389 & 0.724 & 1.476 & 1.923 \\
\hline
\end{tabular}

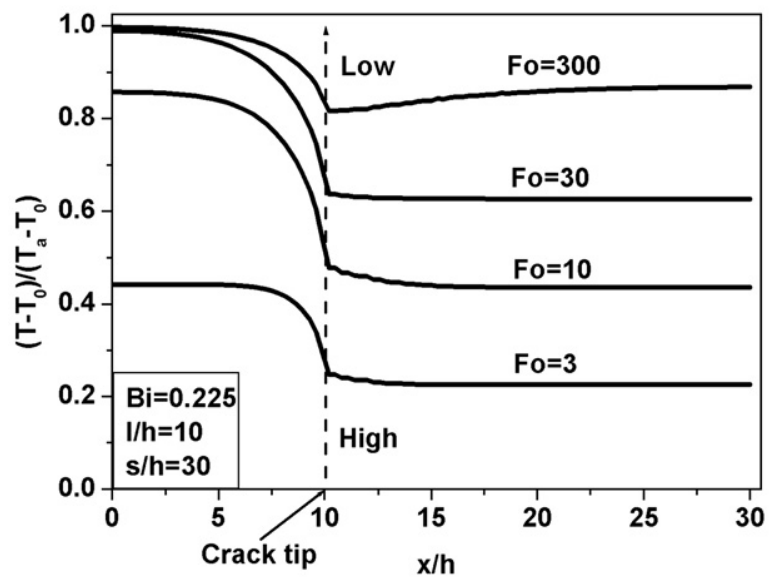

Fig. 6. The normalized transient temperature along the crack plane $y=0^{+}$, $\left(T-T_{0}\right) /\left(T_{\mathrm{a}}-T_{0}\right)$, versus normalized $x$-coordinate, $x / h$, for various dimensionless time instants. $\mathrm{Bi}=0.225,1 / h=10, s / h=30$ and other inputs are listed in Table 1.

and high temperature region of ordinate. The normalized temperature distribution along the crack plane $y=0^{+},\left(T-T_{0}\right) /$ $\left(T_{\mathrm{a}}-T_{0}\right)$, is plotted against normalized $x$-coordinate, $x / h$, in Fig. 6 for various dimensionless time, $\mathrm{Fo}_{\mathrm{o}}=3,10,30,300$. In this plot, the interface crack length is taken as $10 \mathrm{~h}$ and the segmentation crack spacing $30 \mathrm{~h}$. It is shown that, temperature distribution along the interface of coating and substrate is not uniform, reflecting the disturbance effect of insulated interface crack on the temperature field. In overview, the composite medium can be divided into two parts, i.e., the left part with debonded interface and the right part with well-bonded interface. Due to the existence of interface crack, at early time the temperature of the left part drops faster than that of the right part. The heat stored in the left part of substrate has to detour the interface crack before flowing out of the coating. However, after the temperature of coating in the left part is lowered to the ambient temperature, the non-uniformity of temperature tapers down with the elapse of time. Provided convection severity is high enough, this temperature gradient will lead to very high

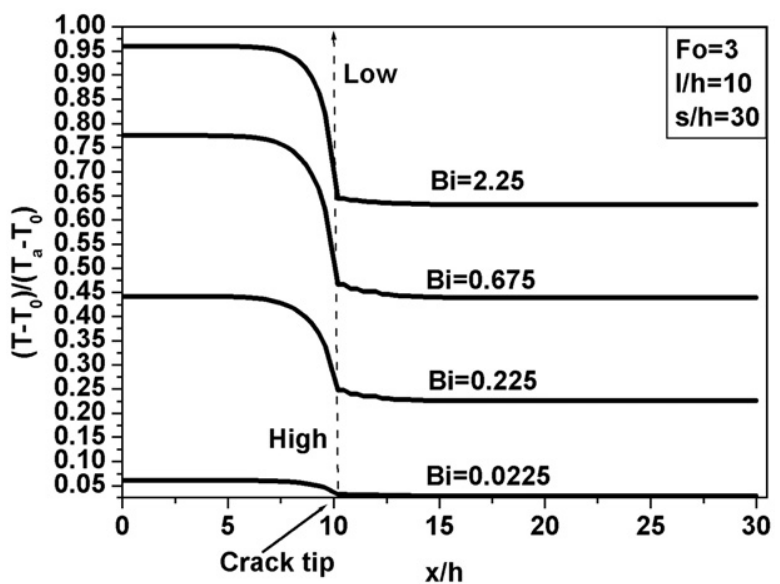

Fig. 7. The normalized transient temperature along the crack plane $y=0^{+}$, $\left(T-T_{0}\right) /\left(T_{\mathrm{a}}-T_{0}\right)$, versus normalized $x$-coordinate, $x / h$, for various convection severity. Fo $=3,1 / h=10, s / h=30$. Other inputs are listed in Table 1. 


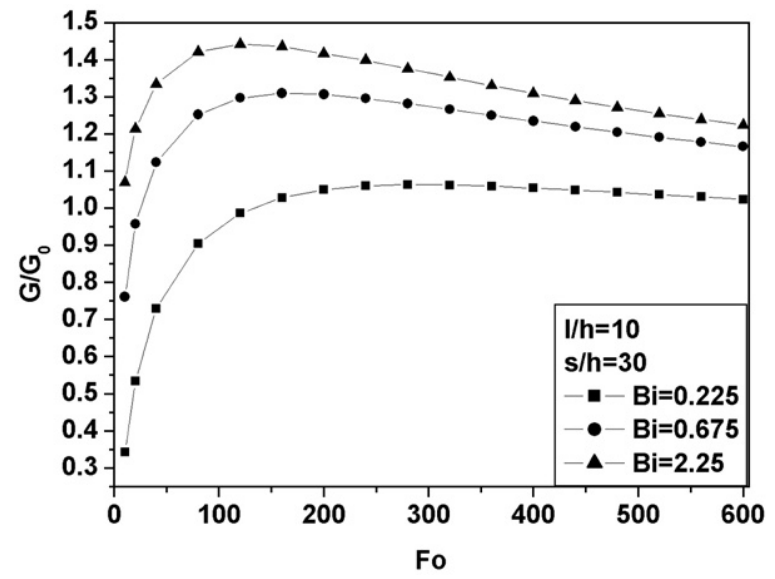

Fig. 8. The normalized energy release rate for interfacial crack, $G / G_{0}$, versus dimensionless time, Fo, for various convection severity. $G_{0}=E_{1}\left(\alpha_{1} \theta_{0}\right)^{2} h, 1 /$ $h=10, s / h=30$. Other inputs are listed in Table 1 .

thermal stress and thus drive the propagation of interface crack. In order to investigate the effect of convection severity on temperature distribution, different Biot numbers $\mathrm{Bi}=$ $0.0225,0.225,0.675,2.25$ are selected in Fig. 7 to find that, the increase of Biot number could significantly sharpen the difference of temperature along the interface at early instant $F_{0}=3$.

\subsection{Results of energy release rate for interfacial crack}

Some results of interfacial crack driving force, represented by energy release rate, are plotted in Figs. 8-11. Firstly, the variation of normalized energy release rate, $G / G_{0}$, versus dimensionless time, Fo, for various Biot number, $\mathrm{Bi}=0.225$, $0.675,2.25$, is plotted in Fig. 8. From it one can see, the energy release rate first increases sharply with time up to the peak value $G_{\max }$, followed by a docile decrease. From point view of linear elastic fracture mechanics (LEFM), an interface crack extension would always stop when the interfacial fracture toughness is high enough to exceed $G_{\max }$. Therefore the most critical mo-

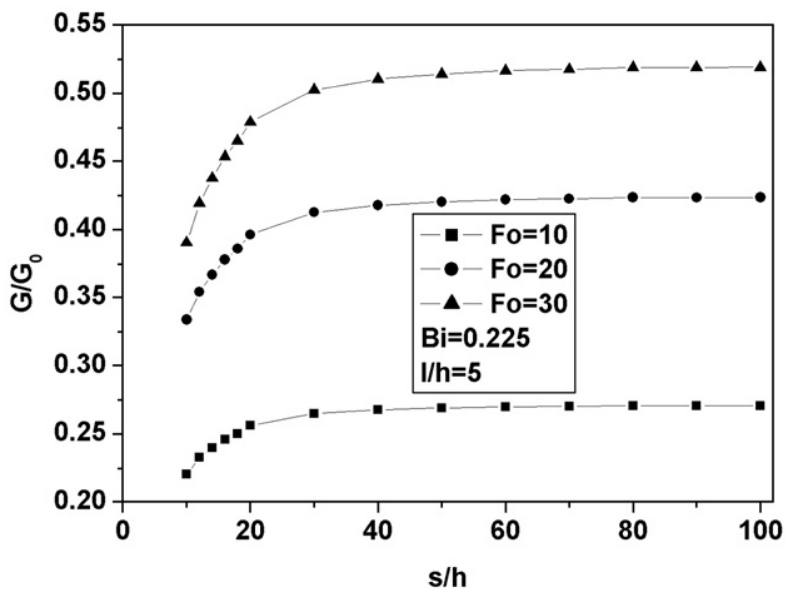

Fig. 9. The normalized energy release rate for interfacial crack, $G / G_{0}$, versus normalized segmentation crack spacing, $\mathrm{s} / \mathrm{h}$, for various dimensionless time. $G_{0}=E_{1}\left(\alpha_{1} \theta_{0}\right)^{2} h, \mathrm{Bi}=0.225,1 / h=5$. Other inputs are listed in Table 1.

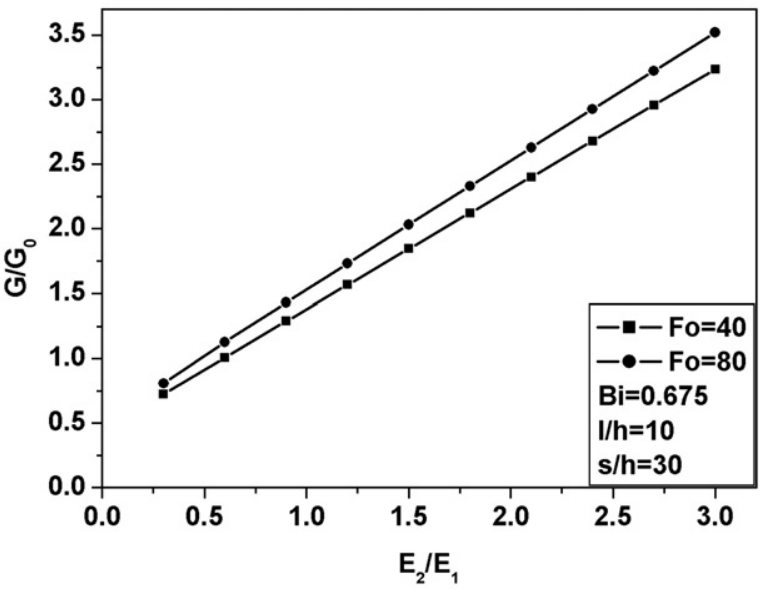

Fig. 10. The normalized energy release rate for interfacial crack, $G / G_{0}$, versus normalized Young's modulus, $E_{2} / E_{1}$, for various dimensionless time. $G_{0}=E_{1}$ $\left(\alpha_{1} \theta_{0}\right)^{2} h, \mathrm{Bi}=0.675,1 / h=10, s / h=30$. Other inputs are listed in Table 1 .

ment the coated medium experience is not at steady state of thermal transient, but at some intermediate instant. This variation trend is in consistent with the corresponding time-dependent temperature distribution described in Fig. 6. Therefore, considering the steady state as the most critical stage will obviously underestimate the severity of thermal transients in practical situation. The influence of convection severity measured by Biot number on the energy release rate can also be induced from Fig. 8. As more severe thermal loading is applied, the magnitude of energy release rate becomes larger and the moment at which the peak value $G_{\max }$ occurs comes earlier.

In Fig. 9 the variation of normalized energy release rate, $G / G_{0}$,versus normalized segmentation crack spacing $\bar{s}=s / h$ for various dimensionless time, Fo $=10,20,30$, is plotted. Since the segmentation crack density is inversely related to the segmentation crack spacing, the graph actually represents the dependence of interfacial delamination driving force on the former. It is shown that, with the decrease of segmentation crack spacing, the magnitude of energy release rate first

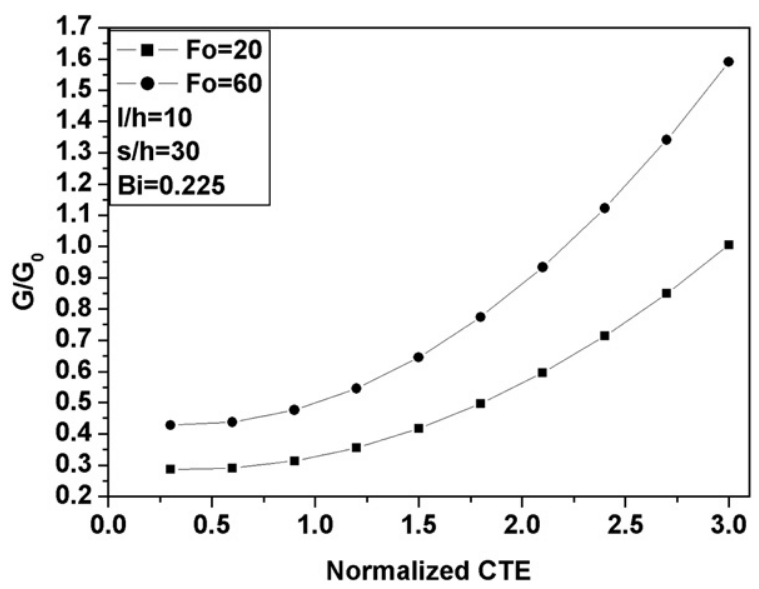

Fig. 11. The normalized energy release rate for interfacial crack, $G / G_{0}$, versus normalized CTE, $\alpha_{2} / \alpha_{1}$, for various dimensionless time. $G_{0}=E_{1}\left(\alpha_{1} \theta_{0}\right)^{2} h$, $\mathrm{Bi}=0.225,1 / h=10, s / h=30$. Other inputs are listed in Table 1 . 
decreases slightly, followed by a steeper decrease. Therefore, the reason why a segmented coating has higher delamination resistance than an intact one is that the former can cause the drop of the energy release rate for interfacial crack propagation. It can also be concluded that until the segmentation crack density is large enough, can this beneficial effect be shown notably. Fortunately the segmentation crack density can be controlled by adjusting the deposition parameters [13]. This change trend can be understood as follows: when the segmentation crack spacing is very large, the interaction between segmentation cracks is little; on the contrary, when the segmentation crack spacing becomes smaller and smaller, the redistribution of thermal stress induced by the introduction of multiple segmentation cracks generates "shedding effect", causing the decrease of stress intensity in the vicinity of the interfacial crack tip.

It is also implied in Fig. 9 that, as the segmentation crack spacing is decreased to a critical extent such that $G_{\max }<\Gamma_{i}$ (Interfacial fracture toughness), the interface crack may be arrested, leaving a ligament of coating still attached to the substrate. This result is in accordance with the well-known "convergent debonding" phenomenon [4], which was usually used to measure the interface fracture toughness of filmsubstrate system.

Finally, the influence of some material constants of interest on the interfacial crack driving force is studied. The effect of $\bar{v}=v_{2} / v_{1}$ is often of secondary consequence for energy release rate and therefore ignored here [1]. Fig. 10. illustrates the effect of Young's modulus ratio $E_{2} / E_{1}$ on the energy release rate, indicating the increase of modulus ratio can cause the increase of crack driving force and the possibility of interface delamination propagation. This effect was previously validated in the case of mechanical loadings by Hutchinson and Suo [3]. It is shown in Fig. 11 that the normalized energy release rate is an increasing function of normalized ratio of coefficients of thermal expansion (CTE) $\bar{\alpha}=\alpha_{2} / \alpha_{1}$. This implies that, for a given substrate, the increase of CTE of the coating may lead to a reduction of the driving force for interface delamination. This dependency could be understood as follows: for a given substrate, the coating with higher CTE has higher capability to accommodate thermal mismatch strain, thus leading to the enhancement of delamination resistance. Furthermore, compared to that of Young's modulus ratio, this dependence trend seems more notable upon further increase of CTE ratio.

\section{Conclusions}

In this paper, the thermally induced interfacial delamination problem of a segmented coating is presented, which is modeled as a coated semi-infinite medium with uniformly spaced segmentation cracks within the coating. Sensitivity analysis is carried out to assess the effect of some important factors on the driving force for interfacial delamination propagation. Results indicate the segmentation crack spacing appears to be an important parameter in the variation of the energy release rate for interface cracks. By decreasing the segmentation crack spacing, the energy release rate firstly becomes slightly smaller in magnitude, then sharply upon further decrease. This confirms that a segmented coating has higher delamination resistance than an intact one under the same thermal transients. The most critical moment the coated medium experience is found to be at an intermediate instant rather than at the steady state of thermal transient. Convection severity measured by Biot number is also quite significant. Not only can it increase the energy release rate, but also the peak value will occur earlier. For a given substrate, the increase of Young's modulus and/or coefficient of thermal expansion of coating are conducive to the reduction of energy release rate for interface cracks, thus helpful for the enhancement of coating delamination resistance.

\section{Acknowledgements}

The financial support provided by the National Natural Science Foundation of China (Grant No. 50471087, 50531060) is greatly acknowledged.

\section{References}

[1] J.W. Hutchinson, A.G. Evans, Surf. Coat. Technol. 149 (2002) 179.

[2] J.H. Underwood, A.P. Parker, G.N. Vigilante, P.J. Cote, ASME J. Press. Vessel Technol. 125 (2003) 299.

[3] J.W. Hutchinson, Z. Suo, Adv. Appl. Mech. 29 (1992) 63.

[4] M.Y. He, A.G. Evans, J.W. Hutchinson, Acta Mater. 45 (1997) 3481.

[5] T. Patterson, D.I. Orloff, Math. Comput. Model. 35 (2002) 165.

[6] F. Spaepen, Acta Mater. 48 (2000) 31.

[7] X. Chen, K. Zhang, G. Chen, G. Luo, Surf. Coat. Technol. 201 (2006) 815.

[8] J.H. Kim, M.C. Kim, C.G. Park, Surf. Coat. Technol. 168 (2003) 275.

[9] S.K. Jha, Y.H. Sohn, S. Sastri, N. Gunda, J.A. Haynes, Surf. Coat. Technol. 183 (2004) 224.

[10] Y.Z. Tsai, J.G. Duh, Surf. Coat. Technol. 200 (2005) 1683.

[11] Y. Hu, K. Zhang, G.N. Chen, C.W. Wu, J. Metal. Treat. (Suppl.) 30 (2005) 161 (in Chinese)

[12] K. Zhang, C.W. Wu, Y. Hu, G.N. Chen, Solid State Phenomena 118 (2006) 243.

[13] G.N. Chen, G.X. Luo, K. Zhang, et al., Acta Armament. (Suppl.) 24 (2003) 6 (in Chinese).

[14] B. Zhou, K. Kokini, Mater. Sci. Eng., A Struct. Mater.: Prop. Microstruct. Process. 348 (2003) 271.

[15] B. Zhou, K. Kokiki, Surf. Coat. Technol. 187 (2004) 17.

[16] E. Sternberg, J.G. Chakravorty, ASME J. Appl. Mech. 26 (1959) 503.

[17] T. Atarashi, S. Minagawa, Int. J. Eng. Sci. 30 (1992) 1543.

[18] T.C. Miller, R. Chona, Eng. Fract. Mech. 59 (1998) 203.

[19] S. Nusier, G. Newaz, Eng. Fract. Mech. 60 (1998) 577.

[20] C. Bjerken, C. Persson, Eng. Fract. Mech. 68 (2001) 235

[21] R.E. Smelser, M.E. Gurtin, Int. J. Fract. 13 (1977) 382.

[22] G.I. Barenblatt, Scaling, Self-similarity, and Intermediate Asymptotics, Cambridge University Press, Cambridge, 1996.

[23] Ansys: Guidance to structural analysis (V.0205), Ansys Inc., 2000.

[24] Q.F. Ma, R.S. Fang, L.C. Xiang, S. Guo, Practical Handbook of Thermophysical Properties, Chinese Agricultural Press, Beijing, 1986 (in Chinese). 